\title{
Laboreal
}

Volume $2 \mathrm{~N}^{\circ} 1$ | 2006

Varia

\section{Acerca de la evolución del concepto de actividad}

Sobre a evolução do conceito de actividade

A propos de l'évolution du concept d'activité

About the evolution of the concept of activity

\section{Pascal Béguin}

Tradutor: Mario Poy

\section{OpenEdition}

\section{Journals}

\section{Edição electrónica}

URL: http://journals.openedition.org/laboreal/13806

DOI: $10.4000 /$ laboreal. 13806

ISSN: 1646-5237

\section{Editora}

Universidade do Porto

\section{Refêrencia eletrónica}

Pascal Béguin, «Acerca de la evolución del concepto de actividad», Laboreal [Online], Volume $2 \mathrm{~N}^{0} 1$ ।

2006, posto online no dia 01 julho 2006, consultado o 10 outubro 2019. URL : http://

journals.openedition.org/laboreal/13806 ; DOI : 10.4000/laboreal.13806

Este documento foi criado de forma automática no dia 10 outubro 2019.

\section{cc) (7) (5)}

Laboreal está licenciado com uma Licença Creative Commons - Atribuição-NãoComercial 4.0 Internacional. 


\title{
Acerca de la evolución del concepto de actividad
}

\author{
Sobre a evolução do conceito de actividade \\ A propos de l'évolution du concept d'activité \\ About the evolution of the concept of activity
}

Pascal Béguin

Tradução : Mario Poy

\section{NOTA DO EDITOR}

Manuscrito recibido en : marzo/2006

Aceptado tras peritage en : junio/2006

El concepto de actividad está emergiendo con fuerza en la escena internacional y en numerosas comunidades. La definición oficial de la ergonomía, adoptada por el consejo de la International Ergonomics Association (IEA, http://www.iea.cc/) en agosto de 2000, afirma que la ergonomía es "a systems-oriented discipline which now extends across all aspects of human activity [1]". Para ese entonces, en el seno de la Sociedad de Ergonomía de Lengua Francesa (SELF, http://www.ergonomie-self. org/), se discutía acerca de la conveniencia de reemplazar los términos "de ergonomía de lengua francesa" por "de ergonomía de la actividad". Destaquemos de inmediato que, naturalmente, adherimos a esa propuesta: los términos "ergonomía de lengua francesa" son vehículo de un patriotismo epistemológico problemático, y cierto es que la ergonomía de la actividad se desarrolla más allá de la francofonía (en particular, en los países de habla portuguesa y española) Más recientemente, y en forma independiente de la ergonomía, se realizó el primer congreso de la nueva Internacional Society for Cultural and Activity Research (ISCAR, http://www.iscar.org), organizado en Sevilla en setiembre de 2005, que reunió a 1700 personas. Los anteriores son sólo tres ejemplos [ ${ }^{2}$ ] de la actual efervescencia en torno a la noción de actividad. ¿Cuáles 
son sus orígenes? ¿Qué nuevos recortes se operan sobre esta noción? ¿Cuáles son las tendencias en juego y sus relaciones?

2 En este texto, intentaremos aportar algunos elementos de reflexión sobre estas preguntas. Por supuesto, y como siempre, sólo se trata de un punto de vista. Mi reflexión, limitada a la ergonomía, se basa en los trabajos llevados a cabo en el Laboratorio de Ergonomía del CNAM [3]. Inicialmente, señalaré el lugar que, según mi parecer, esta noción ha ocupado en dicho laboratorio, como un término que apunta, ante todo, a designar aquello que el trabajo tiene de humano. Sobre esta base, expondré la idea de que la noción de actividad está relacionada con la recuperación de una corriente internacional que había casi desaparecido, pero que está renaciendo. Sin embargo, este resurgimiento puede ser objeto de equívocos. Retomando la distinción propuesta por Cassirer (1910/1991) entre un enfoque sustancialista y uno funcional de los conceptos, fundamentaré la idea de que este desarrollo del concepto de actividad puede obedecer a dos tendencias contradictorias.

\section{El trabajo humano}

3 Desde el punto de vista etimológico, las cosas son simples : el término "ergonomía" significa leyes del trabajo. Pero ¿qué es el trabajo? ¿Se trata de la condición social obrera, del mercado de trabajo, de la transformación de energía, que ya estudiaba Amar (1914) en su obra titulada "El motor humano" ? En un notable texto de síntesis, Teiger (1993) escribía que "el trabajo es una actividad con finalidad, efectuada de manera individual o colectiva, por un hombre o una mujer dados, en una temporalidad determinada, situada en un contexto particular que fija las exigencias de la situación. Esta actividad no es neutra, sino que, a su vez, compromete y transforma a quien la cumple" (Teiger, ibid., p. 79). Planteemos la siguiente hipótesis : en su sentido más amplio, la actividad es lo que distingue al Hombre de la máquina, en el trabajo.

4 Ahora bien, una parte importante (en cuanto a la cantidad) de los trabajos sobre ergonomía se ocupan justamente de la máquina humana, en sus dimensiones psicológicas (la mecánica perceptiva, mnésica, etc.) o fisiológicas (el equilibrio, las regulaciones térmicas, etc.). Así, por ejemplo, en el último número de 2004 de la revista inglesa "Ergonomics" aparecen textos que tratan sobre el impacto "de los protectores auditivos sobre la estabilidad postural", o "del uso de calcetines sobre la temperatura de los pies". Estos saberes generales sobre el "factor humano" no deben ser objeto de una mirada despectiva, ya que definen unas "condiciones límite" más allá de las cuales no es posible ir, sin correr el riesgo de alterar la maquinaria humana. Pero estos conocimientos no son suficientes : las características de la audición no permiten saber que, al operar determinada máquina, el ruido del mecanismo es un índice de primer orden para garantizar la calidad del producto fabricado. Si se cubre la máquina sin tener en cuenta este hecho, la calidad no podrá asegurarse. Esto es inaceptable, incluso para el operador, que trabajará entonces con la cobertura abierta, es decir, sin protección y poniendo en juego su salud.

5 Destaquemos entonces algunas ideas que, a menudo, son evocadas para caracterizar la actividad de trabajo :

- Cuando se habla de actividad, se pone el acento en la persona, en su calidad de actor humano (y no como "factor humano", componente de un sistema). Aun en el caso de las tareas más repetitivas, no se puede hablar de "trabajo manual" o de "trabajo de ejecución" (Teiger \& 
Laville, 1972), ya que el actor humano regula su funcionamiento (Faverge, 1966 ; Leplat, 1971), como así también el sistema en su conjunto, y lo desarrolla (Rabardel \& Pastré, 2005).

- La actividad es siempre singular, dado que caracteriza el trabajo de individuos singulares y variables (singularidades que van desde las dimensiones fisiológicas -por ejemplo, la fatiga-, hasta los aspectos culturales - Wisner, Pavard, Benchekroun \& Geslin, 2003), efectuado en contextos singulares y variables (en sus dimensiones materiales, organizacionales o sociales).

- La actividad tiene finalidad, es decir que tiende hacia un objeto para alcanzar un fin. La construcción de esta finalidad le compete al trabajador : los problemas que los operadores tienen que resolver en su actividad nunca están definidos de manera completa en el enunciado formal de las tareas a realizar, ni son provistos bajo una forma constituida ; por el contrario, ellos deben construirlos (Wisner, 1995). Sin embargo, el trabajo está heterodeterminado, es decir, determinado por los otros. La actividad es el lugar en el que converge la tensión entre el uso de sí por uno mismo y el uso de sí por los otros (Schwartz, 1988).

- Hablar de la actividad de trabajo implica plantearse la hipótesis de que los problemas encontrados en una situación determinada sólo pueden ser identificados a partir de un abordaje "intrínseco" (Theureau, 1992) : la comprensión de la estructura interna de la actividad es la que permite comprender la naturaleza de los problemas, tal como éstos son pensados por los trabajadores. En este sentido, identificar la actividad significa identificar el trabajo real, más que uno prescrito y teórico (Daniellou, Laville \& Teiger, 1983). Desde esta perspectiva, lo real es aquello que se encuentra y se conforma en la actividad (y de lo cual sólo una pequeña parte es observable).

- En su actividad, los trabajadores deben efectuar compromisos entre dos esferas de intereses : los relativos a ellos mismos (la "salud" - física y también psíquica, Dejours, 1988 -, las “competencias" Leplat \& Montmollin, 2001 o las dimensiones subjetivas - Clot, 1999), y los intereses relativos a la producción. Estos intereses pueden ser contradictorios.

- Trabajar es producir una respuesta original, que debe articular y recomponer en la acción un vasto conjunto de determinantes, muchas veces contradictorios. La actividad es "integradora" (Guérin, Laville, Daniellou, Durrafourg \& Kerguelen, 1997). Los determinantes en juego son extremadamente numerosos y no pueden ser reducidos "al puesto". Dimensiones tales como la historia del sujeto (Daniellou, 1998) o las relaciones trabajofuera del trabajo (Curie \& Hajjar, 1987) son, por ejemplo, muy importantes.

6 Además de las referencias indicadas más arriba, el lector interesado puede consultar las siguientes obras : Dejours (1995), Schwartz (1997), Daniellou (1996), Clot (1999), Leplat (2000) o Falzon (2004).

\section{La actividad de trabajo}

7 Pero ¿dónde han encontrado los ergónomos el término actividad ? Puesto que, si bien lo han mantenido con vida, renovándolo (conjuntamente con otros), no son ellos quienes lo han inventado. Por ejemplo, Lahy y Laugier abrían la introducción al primer número de El Trabajo Humano con las siguientes palabras: "La organización racional de la actividad humana plantea problemas teóricos y prácticos de extrema complejidad, cuyo estudio requiere la colaboración de ciencias y técnicas muy variadas". Estábamos en 1933 y la ergonomía no existía. Pero es cierto que una palabra no es suficiente para definir un concepto, y que un mismo concepto puede esconderse detrás de diferentes 
términos. En numerosos textos, encontraremos más bien el término "conducta". Y, de hecho, la palabra actividad es un término habitual del francés [4], cuyo uso puede producir equívocos [5]. genealogías. Sin embargo, esta diversidad léxica es sólo una de las dimensiones, y quizás no la más importante, entre aquellas a tener en cuenta cuando se trata de investigar la génesis de esta noción. En una obra reciente, Bronckart et al. (2004) nos dan algunas pistas. "La sociología, la psicología, la lingüística y las ciencias de la educación han surgido sobre un fondo de debates complejos, relacionados con los modelos de referencia (¿se trata de ciencias naturales, de la mente, socioculturales ?), así como con la delimitación y definición de las unidades de análisis (comportamiento, conductas, representaciones, signos, hechos sociales, etc.) y con los procedimientos metodológicos (observación, experimentación, introspección, hermenéutica, etc.). En este contexto general, y a través de las obras de Bühler, Clarapède, Dewey, Durkheim, Saussure, Vygotski y muchos otros, se constituyó un vasto movimiento transversal que ponía el acento en la unidad del objeto de las ciencias humanas, y que preconizaba, por lo tanto, la articulación de estas disciplinas en el marco de una "ciencia de la mente y de la sociohistoria" [...]. A partir de los años '30, sin embargo, este movimiento fue combatido, disminuido, y luego casi desaparecido, bajo el efecto del surgimiento de ciertas corrientes que reivindicaban la total autonomía e impermeabilidad de cada una de las ciencias humanas, y que se anclaban, entonces, en el positivismo o en su derivado, el estructuralismo." (op. cit. p13-14).

Comparto este diagnóstico y quisiera presentar en este texto la idea de que será un fermento intelectual (científico, filosófico y político) sumamente difuso el que constituirá el terreno a partir del cual emergerá, en la ergonomía de lengua francesa, una cierta visión del trabajo humano, que se designará como "actividad de trabajo". Algunos autores son conocidos en ergonomía (aunque no necesariamente leídos), como H. Wallon, G. Canguilhem o S. Weill. Luego, hay otros, casi olvidados, cuya influencia no es menor. Entre ellos, me limitaré a evocar a uno solo, a modo de ejemplo.

\subsection{Ignace Meyerson}

Meyerson publicó una única obra : "Las funciones psicológicas y las obras". La editorial Presses Universitaires de France (PUF) ha publicado recientemente una compilación de sus textos, provenientes principalmente de la revista que él dirigía, "le journal de psychologie normale et pathologique". Si bien este autor no utilizaba el término "actividad", creo que fue un teórico de este concepto. A continuación, destacaré siete puntos :

- Meyerson insiste, en primera instancia, sobre el carácter sistemático de las conductas humanas. Los actos de los hombres se organizan en función de su objetivo, de las normas sociales, de imperativos reconocidos como tales. Esto imbrica a las conductas humanas en sistemas simbólicos culturales -lenguaje, religión, jerarquías sociales. En una palabra, la acción no puede comprenderse sin hacer referencia al sistema simbólico al que está subordinada.

- Las conductas humanas están orientadas, en primer lugar, por el trabajo, que es productor de objetos o valores juzgados como necesarios o deseables por el grupo con el cual el 
individuo se identifica, productor, en definitiva, del mundo simbólico en el cual el hombre vive.

- Las conductas humanas no son pasivas ni constituyen reacciones: son activas y experimentales, en el sentido de que están reguladas por las relaciones entre medios y fines. El hombre no es sensible solamente a los efectos de sus propias acciones, sino también a los de las acciones de otros. Está tan atento a las intenciones que gobiernan los actos, como a los resultados.

- Las conductas humanas están orientadas por la construcción de un trabajo, de una obra: "el hombre es fabricante y creador". Es por ello que "el espíritu es más espíritu cuando está realizado" y que "la obra crea al espíritu, al mismo tiempo que lo realiza".

- Podemos decir que el Hombre está inmerso en sus obras : lenguas, religiones, leyes, ciencias, medios de producción. Ellas son quienes le dan forma. Este mundo de obras, que constituye la historia de la civilización, también constituye la historia del funcionamiento psicológico del ser humano.

- Las obras se diferencian en una variedad de esferas dentro de una misma cultura y de una cultura a otra. Cada una de estas esferas está determinada por su particular lenguaje y sistema de símbolos, los cuales evolucionan con la historia y el ingenio de los hombres.

- El hombre está orientado hacia el futuro, "haciendo...". Realiza sus construcciones con la mirada puesta en los resultados futuros, teniendo en mente no las piedras con las cuales construye, sino la casa que serán. Los grandes proyectos colectivos refuerzan esta orientación hacia el futuro. Pero las concepciones de aquello que el futuro debe ser y de las acciones que, en consecuencia, hay que emprender son tan divergentes dentro de una misma cultura que de esto se desprenden conflictos y tensiones. La armonización de estas diferentes representaciones del futuro constituye un problema humano fundamental, y uno central en la historia.

\subsection{Regreso a nuestro futuro}

11 Llegado a este punto de mi exposición, podría ser yo acusado (con todo derecho) de plagio, lo cual es grave para un docente universitario. La presentación de Meyerson que acabo de hacer es una copia, casi palabra por palabra (sustrayendo los desarrollos), de una parte de un texto escrito por un americano : J. Bruner (Bruner, 1996, pp. 200 a 203). Espero que se perdone mi delito, ya que éste apunta, ante todo, a agradecer al autor plagiado. Gracias, señor Bruner, por haber evocado la obra de este gran autor.

No obstante, me pregunto : ¿cómo es que un psicólogo de habla francesa, considerado como un autor de referencia por un gran investigador americano, no me ha sido propuesto durante mis años de estudio de psicología, mientras que sí he sido obligado a aprender de memoria las ideas de aquellos que se ocupaban del funcionamiento de la paloma! Es verdad que Meyerson no ha sido nunca totalmente olvidado por quienes se han ocupado del estudio del trabajo (por ejemplo : Curie \& Guillevic, 1979; Clot, 1999 ; Leplat, 2000). Pero no es allí donde se ha desarrollado la tendencia principal. El compendio de M. Reuchlin (1977), que era la obra de referencia en la época en la cual yo era estudiante de psicología, sólo evoca una vez a Meyerson... ¡En el capítulo consagrado a la percepción!

Bruner propone una respuesta : "Hace ya un siglo que la psicología se ha transformado en una disciplina académica : es, por lo tanto, respetable. Pero la respetabilidad se paga [...] ¿Cómo interpretamos el mundo, cómo lo desciframos, cómo elaboramos las significaciones?, se trata de interrogantes que no podían constituirse en temas de 
estudio adecuados mientras no se lograra traducirlos a magnitudes manipulables, tales como centímetros, gramos, segundos, probabilidades. Como consecuencia de esta negociación "fáustica" [...] el proceso de adaptación podía ser estudiado en condiciones controladas de laboratorio, sin preocuparse por el transcurso desordenado de los hechos en el mundo real" (Bruner, op. cit., pp. 193-194).

Podemos tener confianza en el diagnóstico del autor. Jérôme Bruner ha sido, no es necesario subrayarlo, el padre de la revolución cognitiva aunque, hoy en día, defiende otra revolución. Pero hoy en día el autor preconiza otra revolución, de la cual es probable que el concepto de actividad no sea independiente. Lo que rescato como enseñanza de la lectura de Bruner es, por lo tanto, lo siguiente : la ergonomía de la actividad constituye más nuestro futuro que nuestro pasado. El trabajo a realizar está por delante de nosotros, y no por detrás. Lo cual no significa que la lectura de antiguos autores o de aquello que ya fue capitalizado no sirva. Queda por realizar un trabajo de genealogía que permita evaluar mejor los avances realizados, así como relacionar las diferentes tradiciones. Se trata pues de un trabajo grande y muy difícil, que sólo tendrá sentido en la medida en que nos ayude a procesar mejor y aprehender los problemas que se plantean en la actualidad. Por el contrario, hay que regresar a nuestro futuro, y la comunidad debe darse las herramientas necesarias para facilitar este trabajo. Diversas revistas, como Plureal [6] o @ctivités, tienen seguramente un papel más que importante en este contexto.

\section{3. ¿Ha dicho usted "concepto" ?}

Resulta entonces indispensable un trabajo conceptual. Como hemos señalado, el término "actividad" es una noción bastante amplia e imprecisa y, tal vez, lo suficientemente vaga como para que estemos todos de acuerdo. Esto tiene la ventaja de mantener la identidad de una comunidad, pero dudo de que este acuerdo superficial resulte productivo. Una gran parte de los textos de los ergónomos de lengua francesa acerca de la actividad se asocia con la "acción situada" (como lo ha señalado Wisner en 1995). En otros trabajos, el trasfondo del término " actividad » se sitúa en la psicología cognitiva computacional. Esto denota quizás una cierta riqueza, pero es necesario sin embargo tener cuidado : abriendo demasiado el sentido, corremos el riesgo de vaciar a las reflexiones de su contenido. En mi opinión, hay que fijar una doble frontera. Por un lado, nos alejamos de la actividad cuando lo que se busca captar es el trabajo del trabajador únicamente a nivel del "sujeto", como lo hace la psicología cognitiva. Por otro lado, también nos alejamos de ella cuando se busca ubicarla dentro de las características de las situaciones, sólo en sus dimensiones artefactuales $\mathrm{u}$ organizacionales, como lo postula la acción situada (Béguin \& Clot, 2004). Pero señalemos que esta doble frontera constituía uno de los puntos argumentados en la obra fundadora de Ombredane et Faverge (1955) : no hay que estudiar al trabajador y a la tarea separadamente, sino la actividad de trabajo en sí misma. Esta posición ha sido reafirmada recientemente por Leplat (2000) : la actividad es un acoplamiento entre la tarea y el sujeto. Comparto esta posición, aun si pienso que el sujeto y la tarea mantienen relaciones dificultosas, mucho más difíciles, en todo caso, de lo que ciertos enfoques de la acción situada permiten suponer, al postular un acoplamiento casi automático, a través del concepto de affordance [ $\left.{ }^{7}\right]$. Por el contrario, el análisis del trabajo nos ha mostrado que, a menudo la tarea se vuelve a poner en movimiento en la 
actividad del trabajador. Además, el análisis del trabajo de los diseñadores ha mostrado que, detrás de la tarea lo que encontramos es la actividad del diseñador.

Sin embargo, un trabajo sobre el concepto de actividad no deja de generar interrogantes. Un reciente debate sobre las relaciones entre formación, ergonomía y análisis del trabajo, organizado por la revista "Education permanente", se hizo eco de la cuestión [8]. En él, Schwartz recordó que la actividad es un concepto profundamente enigmático, sobre el cual nadie puede pretender hacer teoría. Subraya el riesgo de que investigadores, formadores o ergónomos partan de tal o cual teoría para aprehender la realidad de los medios en los cuales se interesan. En respuesta, Clot argumenta que se puede desarrollar una especie de abstracción sobre la actividad. Así, "con demasiada frecuencia actualmente la teoría de la actividad se torna una especie de máquina de fabricar conceptos, $\mathrm{y}$, algunas veces, hasta coloquios".

Se trata de un debate fundamental. Por un lado, una comunidad no puede ubicar en el centro de sus intercambios un concepto sin saber definirlo. Por otro lado, estos debates y ambiciones conceptualizadoras y modelizantes presentan el riesgo de un nuevo one best way [ [9]. La contradicción es profunda pero, a mi entender, suscita sobre todo una reflexión sobre el estatus de los conceptos y de los procesos de conceptualización en general.

Como lo ha mostrado Cassirer (op. cit.), hay dos modos de aprehender lo que es un concepto. El primero es pensar que un concepto busca captar una sustancia. Las cosas que vemos son objetos con propiedades definidas y tejen relaciones determinadas por leyes. La conceptualización es entonces una abstracción que evoluciona hacia una mejor captación de una realidad que ya está allí. El segundo es pensar que un concepto se inscribe en el marco de una función, y de la actividad con finalidad de quien lo trabaja, lo produce o lo utiliza. En este segundo esquema, la producción científica no consistirá tanto en manipular conceptos genéricos que evolucionan continuamente hacia una mejor captación de sustancias, sino en efectuar una construcción funcional que se inscriba en el marco de una actividad con finalidad. Ahora bien, me parece que uno de los puntos interesantes del trabajo filosófico que realiza Schwartz (1988), es justamente el de precisar el estatus funcional de un concepto. Un concepto es un mediador. Por una parte, está destinado a ser un recurso de los protagonistas, y puede, en este sentido, presentar una función emancipadora y ofrecer nuevas potencialidades. Pero, por otra parte, su eficacia supone que sea fecundado por la vida, puesto en movimiento en los medios singulares a los cuales está destinado. Se ponen, entonces, en juego una tensión y un movimiento entre, por un lado, las "normas antecedentes" y, por otro, un trabajo de re normalización.

Este esquema es extremadamente rico. Destaquemos rápidamente tres puntos :

- Las conceptualizaciones producidas por el investigador o el consultor no tienen tanto la finalidad de ir hacia un mayor grado de abstracción, como de producir recursos para la actividad con finalidad del consultor, así como también recursos para la actividad de los protagonistas sobre quienes se efectúa el análisis.

- Se plantea entonces, de manera indisociable, la cuestión del desarrollo de las actividades individuales y colectivas, pero también de los conceptos, dado que ambos polos evolucionan conjuntamente.

- Asimismo, se plantea la cuestión de las formas institucionalizadas (incluida la investigación misma) que están en juego en los procesos de "re normalización", para retomar el término de Schwartz. Foucault (2004) planteó una distinción entre dos formas de dispositivos. Los 
primeros, que él denomina de "normativización", se caracterizan por el hecho de que el saber se transforma en poder, convirtiéndose entonces en la norma -y aquello que no se inscribe en él queda en la anormalidad. El segundo dispositivo, de "normalización", consiste en construir curvas de desarrollo de los saberes para constituir, localmente, la normalidad. ¿Cómo favorecer los dispositivos de normalización, tanto en el plan de investigación, como en la intervención ? En muchos sentidos, es esta dimensión funcional la que caracteriza, en la ergonomía de lengua francesa, a aquello que se designa como actividad de trabajo. Una característica esencial de toda intervención ergonómica es que ésta no se contenta con producir un conocimiento sobre el trabajo humano (Daniellou \& Béguin, 2004). Éste último es, sin lugar a dudas, un objeto de conocimiento, pero este conocimiento apunta a la acción (Teiger, 1993; Guérin et al., 1997). Por otro lado, el análisis de la actividad se realiza asociando a los actores, ya que se trata de una "coproducción" que sólo vale en la medida en que se inscribe en el marco de los intercambios producidos dentro de comunidades heterogéneas, cuyas lógicas pueden ser contradictorias (de Terssac, 1990).

\section{Conclusión}

20 Postular un enfoque funcional de un concepto implica pensar que éste no es independiente de la historia de las comunidades que lo elaboran, ni de las finalidades perseguidas por aquellos que lo utilizan. Y, evidentemente, lo que es válido para los conceptos en general, también lo es para el concepto de actividad.

¿No podríamos concluir, entonces, con la idea de que el desarrollo actual del concepto de actividad es una evidencia del surgimiento de nuevos problemas ? Efectivamente, es la idea que defenderemos. Tres tendencias históricas, cuyos orígenes son muy heterogéneos, se conjugan para suscitar el desarrollo del concepto de actividad.

Una primera tendencia, situada a nivel internacional, y que ya he evocado a través de Bronckart y col. y de Bruner, reside acaso en un progresivo desvanecimiento de los enfoques positivistas. Estos, predominantes durante mucho tiempo, han llegado tal vez a un punto de inflexión en su historia. No obstante, mi visión de los movimientos internacionales es muy parcial, y esta hipótesis sólo podría ser confirmada en un futuro, por historiadores de las ciencias (que yo no soy). Me parece, sin embargo, que lo que actualmente se designa en la escena internacional bajo el rótulo de "teorías de la actividad" es un campo incierto y de gran diversidad, en el que se plantea la cuestión del concepto de actividad no como sustancia, sino como función. Esta es, en todo caso, la esperanza que uno puede abrigar $\mathrm{y}$, al mismo tiempo, una apuesta en el plano internacional. En esta perspectiva, se ha creado un Technical comité $\left[{ }^{10}\right]$ dentro de la IEA, llamado "Activity theory for work analysis and Design" [11].

A este movimiento internacional hay que agregarle las interrogaciones frecuentes que provienen de la ergonomía misma, en Francia. Los ergónomos de lengua francesa han tenido, en la última década, una importante evolución profesional, en la que tienden, cada vez más, a pasar de ser analistas del trabajo a ser diseñadores. ¿Pero entonces el ergónomo acabaría siendo un prescriptor? ¿Y qué es lo que tendría que prescribir, cómo, a quién [12] ? En cierta forma, la ergonomía debe hoy encontrar respuestas a las preguntas que hasta ayer les planteaba a los ingenieros. Podemos pensar que se trata de un movimiento profundo, que conduce a revitalizar y precisar el concepto de actividad.

Finalmente, también aparecen en Francia interrogantes más amplios sobre las dinámicas de cambio. Por un lado, los discursos políticos recurrentes que afirman que 
es imposible transformar un país con un pasado glorioso pero con un porvenir sombrío. Por otro lado, la constatación de los daños causados por las dinámicas desenfrenadas de cambio en los medios profesionales (Le Goff, 1999), en un contexto supuestamente alejado del esquema taylorista (Béguin, Le Joliff \& Vidal-Gomel, 2001), en el que la modernización se negocia en beneficio de la polivalencia, la flexibilidad y la "empleabilidad". No es trivial comprobar, en este contexto, que una gran parte de los debates actuales sobre el concepto de actividad gira justamente en torno a su desarrollo : ¿cómo se desarrolla la actividad de trabajo, cuáles son sus orígenes y sus dinámicas? Se trata de una cuestión de actualidad, y no solamente de una dimensión sustancial de la actividad.

Es cierto, sin embargo, que ésta es sólo una forma de ver las cosas. El concepto de actividad puede inscribirse también en otro universo epistemológico. Se puede intentar aprehender la actividad como una sustancia. En ese caso, el renacimiento actual del concepto de actividad debería interpretarse como un desarrollo de la abstracción conceptual destinada a restablecer aquello que las conceptualizaciones anteriores dejaban en la incertidumbre. Habría entonces que precisar en qué se distingue la finalidad de este movimiento, conducido por iniciativa de los investigadores esclarecidos, de una organización científica del trabajo. En realidad, es probable que esta idea esté elaborándose en los desarrollos actuales del concepto de actividad; sin embargo pienso, por mi parte, que un desarrollo sustancialista del concepto de actividad no aportará nada realmente novedoso.

No obstante, entre estas dos vías de evolución del concepto de actividad, la historia aún no está escrita.

Quisiera agradecer a aquellas personas que han aportado sus remarcas y comentarios a las múltiples versiones preliminares de este artículo, sobre todo a T. Aletcheredji, A. Kerguelen, C. Teiger, Y. Schwartz, y F. Daniellou (quienes, obviamente, no son responsables de las opiniones aquí expresadas), así como a M. Poy, quien ha realizado la traducción.

\section{BIBLIOGRAFIA}

Amar, J. (1914). Le moteur humain. Paris : H. Dunod \& E. Pinat editeurs.

Béguin, P. \& Clot, Y. (2004). L'action située dans le développement de l'activité, @ctivités, 1, (2), 27-49. http://www.activites.org/v1n2/be-guin.fr.pdf

Béguin, P., Le Joliff, G. \& Vidal-Gomel, C. (2001, October). Quelles évolutions des conditions de travail dans un contexte de reengeniering. Proceedings of the joint conference SELF-ACE 2001 "Ergonomics for changing work". Montréal, Canada.

Bronckart, J.P., Bulea, E., Fillietaz, L., Fristalon, I., Giger, I.P. \& Revaz, F. (2004). Agir et discours en situation de travail. Cahiers de la section des sciences de l'éducation, $\mathrm{n}^{\circ} 103$. 
Bruner, J. (1996). Meyerson aujourd'hui : quelques réflexions sur la psychologie culturelle. In Françoise Parot (Ed.) Pour une psychologie historique. Écrit en hommage à Ignace Meyerson (pp 193-207). Paris : PUF.

Cassirer E. (1910, 1991). Substances et fonctions. Paris : éditions de Minuit.

Clot, Y. (1999). La fonction psychologique du travail. Paris : PUF.

Curie, J. \& Guillevic, C. (1979). Personnalisation, changement sociaux et travail. Psychologie et éducation, III/3, 37-50. [Retomado en Curie (2000). Travail, personnalisation, changement sociaux. Archives pour une histoire de la psychologie du travail. Toulouse : Octarès].

Curie, J. \& Hajjar, V. (1987). Vie de travail, vie hors travail. La vie en temps partagé. In Cl. LévyLeboyer \& J. C. Spérandio (Eds.), Traité de Psychologie du Travail (pp. 37-55). Paris : PUF.

Daniellou, F. \& Béguin, P. (2004). Méthodologie de l'action ergonomique, approches du travail réel. In Pierre Falzon (Ed.), Ergonomie (pp. 335-358). Paris : PUF.

Daniellou, F. (1998). Evolution de l'ergonomie francophone : théories, pratiques, et théories de la pratique. In Dessaigne M.F. \& Gaillard, I. (Eds.), Des évolutions en Ergonomie (pp 37-54).

Toulouse : Octarès.

Daniellou, F. (1996). Questions épistémologiques soulevées par l'ergonomie de conception. In F. Daniellou (Ed.), L'Ergonomie en quête de ses principes (pp. 183-200). Toulouse : Octarès.

Daniellou, F., Laville, A. \& Teiger, C. (1983). Fiction et réalité du travail ouvrier. Cahiers Français de la Documentation Pédagogique, 209, 39-45.

Dejours (1995). Le facteur humain. Paris : PUF.

Dejours, C. (1988). Plaisir et souffrance dans le travail. Tomes I \& II. Édition de l'AOCIP.

Falzon, P. (2004). Ergonomie. Paris : PUF.

Faverge, J.M. (1966). L'analyse du travail en termes de régulation. In J.M. Faverge (Ed.),

L'ergonomie des processus industriels (pp. 33-60). Bruxelles : ULB.

Foucault, M. (2004). Sécurité, Territoire, Population : Cours au Collège de France, 1977-1978.

Paris : Hautes Etudes / Gallimard / Seuil.

Guérin F., Laville, T., Daniellou, F., Durrafourg, J. \& Kerguelen, A. (1997). Comprendre le travail pour le transformer, la pratique de l'Ergonomie. (2 ${ }^{\mathrm{a}}$ Ed.) Lyon : Ed. de l'ANACT.

Lahy, J.M. \& Laugier, H. (1933). Avant-propos. Le travail Humain, 1, 1, 1-2.

Le Goff, J.P. (1999). Les illusions du management. Paris : La Découverte. Leplat, J. \& Montmollin, de M. (2001). Les compétences en Ergonomie. Toulouse : Octarès.

Leplat, J. (2000). L'analyse psychologique du travail en ergonomie. Toulouse : Octarès.

Leplat, J. (1971). Planification de l'action et régulation et régulation d'un système complexe.

Bulletin de Psychologie, $n^{\circ}$ 298, 533-538.

Meyerson, I. (1948). Les fonctions psychologiques et les œuvres. Paris : Vrin.

Meyerson, I. (1987). Écrits 1920-1983. Pour une psychologie historique. Paris, PUF.

Ombredane, A. \& Faverge, J-M. (1955). L'analyse du travail. Paris : PUF. Rabardel , P. \& Pastré, P. (2005). Modèles du sujet pour la conception, dialectiques activités développements. Toulouse : Octarès.

Reuchlin, M. (1977). Psychologie. Paris : PUF. 
Schwartz, Y. (1988). Éxperience et connaissance du travail. Paris : Messidor

Schwartz, Y. (1997). Reconnaissance du travail, pour une approche ergologique. Paris : PUF.

Teiger, C. (1993). L'approche ergonomique : du travail humain à l'activité des hommes et des femmes au travail. Education permanente, $n^{\circ} 116,3$, 71-96.

Teiger, C. \& Laville, A (1972). Nature et variations de l'activité mentale dans des tâches répétitives : essai d'évaluation de la charge de travail. Le travail Humain, 35 (1-2), 99-116.

Terssac, de, G. (1990). Impact de l'analyse du travail sur les relations de travail. In M. Dadoy et al. (Eds), Les analyses du travail, enjeux et formes (pp. 27-41). Paris : CEREQ, Collection des Etudes, $\mathrm{n}^{\circ} 54$.

Theureau, J. (1992). Le cours d'action : analyse sémio-logique. Essai d'une anthropologie cognitive située. Berne : Peter Lang.

Wisner, A., Pavard B., Benchekroun H. \& Geslin Ph. (2003). Anthropotechnologie. Vers un monde polycentrique. Toulouse : Octarès.

Wisner, A. (1995). Understanding problem building : Ergonomic Work Analysis. Ergonomics, 38, (8), 1542-1583.

\section{NOTAS}

1. $\mathrm{N}$ del T: En inglés en el original.

2. Pero que conciernen a asociaciones internacionales que reúnen a varias centenas, o aun a varios miles de miembros.

3. En el que primero he efectuado una tesis bajo la dirección de A. Wisner, y donde soy actualmente docente investigador.

4. $\mathrm{N}$ del T: Igual que en español.

5. Se puede hablar, por ejemplo, de actividad económica de las empresas.

6. N. de R: Mientras renombrada Laboreal, conforme explicación en el Editorial del ㄴo número de la Revista (http://laboreal.up.pt)

7. $\mathrm{N}$ del T: En inglés en el original.

8. Este debate, sostenido por Y. Schwart, Y. Clot, F. Daniellou y P. Mayen, ha sido publicado en el número 165 (2005) de la revista "Education permanente", pp. 139-160.

9. $\mathrm{N}$ del T: En inglés en el original.

10. $\mathrm{N}$ del T: En inglés en el original.

11. $\mathrm{N}$ del T: En inglés en el original.

12. Se pueden encontrar elementos de discusión sobre esta cuestión en Daniellou (1996) y en las actas del congreso de la SELF, realizado en 2002 en Aix, sobre el tema de la evolución de la prescripción. (http://www.ergonomieself.org/actes/congres2002.html) 


\section{RESUMOS}

O objectivo deste artigo é o de aportar alguns elementos de reflexão acerca das actuais evoluções do conceito de actividade, a partir de um enfoque ancorado na ergonomia. Por conseguinte, trata-se de recordar que os termos "actividade de trabalho" têm sido utilizados em ergonomia para designar aquilo que é especificamente humano no trabalho, desenvolvendo-se a ideia de que os debates actuais sobre o conceito de actividade constituem uma re-actualização de um enfoque internacional que tinha quase desaparecido, mas que actualmente está a ressurgir. Partindo desta base, distinguem-se duas vias possíveis de desenvolvimento do conceito de actividade. Uma via substancialista, que será criticada, e uma via funcional, destinada a oferecer ferramentas aos actores. De acordo com este último enfoque, os desenvolvimentos e os debates em torno do conceito de actividade dão conta da emergência de questões cruciais em ergonomia.

El objetivo de este artículo es aportar algunos elementos de reflexión acerca de las actuales evoluciones del concepto de actividad, a partir de un enfoque anclado en la ergonomía. Luego de recordar que los términos de "actividad de trabajo" han sido utilizados en ergonomía para designar aquello que es específicamente humano en el trabajo, se desarrolla la idea de que los debates actuales sobre el concepto de actividad constituyen una re actualización de un enfoque internacional que había casi desaparecido, pero que actualmente está resurgiendo. Partiendo de esta base, se distinguen dos vías posibles de desarrollo del concepto de actividad. Una vía sustancialista, que será criticada, y una vía funcional, destinada a brindar herramientas a los actores. De acuerdo con este último enfoque, los desarrollos y los debates en torno al concepto de actividad dan cuenta de la emergencia de cuestiones cruciales en ergonomía.

L'objectif de ce texte est de fournir quelques éléments de réflexion sur les évolutions actuelles du concept d'activité, à partir d'un point de vue situé en ergonomie. Après avoir rappelé que les termes “activité de travail» ont été utilisés en ergonomie pour désigner ce qui est spécifiquement humain dans le travail, on avance l'idée que les débats actuels sur le concept d'activité constituent une reprise d'un courant international qui avait quasiment disparu, mais qui est en train de renaître. Néanmoins, ce développement n'est probablement pas homogène. On distingue deux voies possibles du développement du concept d'activité. Une voie substantialiste, qui est critiquée. Et une voie fonctionnelle, qui vise à outiller les acteurs. Selon cette seconde perspective, les évolutions et les débats actuels autour du concept d'activité témoignent de l'émergence de questions vives en ergonomie.

This article's objective is to shed light on some elements of reflection about the current evolutions in the concept of activity, from an ergonomic perspective. Therefore, it is about reminding that the terms "work activity" have been used in ergonomics to designate what is specifically human in work, developing the idea that the current debates on the concept of activity constitute a re-actualization of an international focus that had almost disappeared, but is emerging again. From this basis, two possible paths of development of the concept of activity are distinguished. A substantial path, that will be criticised, and a functional path, destined to give tools to the actors. According to this last focus, the developments and debates around the concept of activity show the emergence of crucial questions in ergonomics. 
ÍNDICE

Mots-clés: activité, travail, concept, substance, fonction

Palabras claves: actividad, trabajo, concepto, sustancia, función

Keywords: activity, work, concept, substance, function

Palavras-chave: actividade, trabalho, conceito, substância, função

\section{AUTORES}

\section{PASCAL BÉGUIN}

Conservatoire National des Arts et Métiers (CNAM) 41, rue Gay Lussac, 75005 Paris, France

beguin@cnam.fr 